\title{
EQUATION OF AHP AND LOGIT-MODEL AND APPLICATION OF THE RESULTS TO MEASURING OF INFORMATION TECHNOLOGY INVESTMENT
}

\author{
Toshimasa OZAKI ${ }^{1}$, Shigeo YAMAMOTO ${ }^{2}$ \\ ${ }^{1}$ Faculty of Commerce, Nagoyagakuin University , 1350 Kamishinano-Chyo, Seto 480-2398 Japan \\ ozaki@ngu.ac.jp \\ ${ }^{2}$ Information Communication Research Center, Kansai Research Institute, Chudoji Minami-machi, \\ Shimogyo-ku, Kyoto 600-8813 Japan \\ s-yama@kyoto.kansai-ri.co.jp
}

Keywords: information entropy, utility function, Markov chain

\begin{abstract}
Summary: AHP and Logit-model were theoretically studied as typical decision-making models, and the consideration of the relations and the use of the result were mentioned in this paper. In the first half of the paper, it was shown that the pair matrix of AHP can be approximated as a transition matrix by steady-state Markov chain when it is assumed that evaluation value in AHP is utility function. Furthermore, by introducing information entropy and linear form of utility function, information entropy of AHP can be maximized, and the expected values of AHP reaches a maximum as with Logit-model. And the results show that AHP can become the same decision-making model as Logit-model. On the other hand, it was shown that equation of AHP and Logit-model could be applied as a technique to measuring of information technology investments in computer hardware, software and so on. These results not only show that AHP as a qualitative decision-making tool complements the Logit-model, but also clarify that information technology investment contributes to productivity in organizations.
\end{abstract}

\section{Introduction}

The AHP model proposed by Saaty is a typical decision-making model. On the other hand, Logit-model, used extensively for the course choice in traffic engineering, is also a typical tool used as a decision-making model. However, these decision-making models have been developed independently, and virtually no researcher has studied the relations between the two models. In the first half of this paper, the position is taken that the evaluation values in AHP are the utility values, and relationships between AHP and Logit-model are examined by using information entropy that describes uncertainty.

Incidentally, there remains a problem ${ }^{(1)-(3)}$ of quantification of the effect of investments on computer hardware and software, or so-called information technology investments. An appropriate methodology for measuring the effects has eagerly been sought for globally. The latter half of this paper describes a measurement technique, which has been developed on the basis of the relations between AHP and Logit-model identified in the first half.

\section{Interpretation of AHP and Logit-model with the utility value}

\subsection{Correlation between AHP and Logit-model ${ }^{(4)}$}

We have made it clear that the utility value of Logit-model can be estimated from the synthetic evaluation value about the alternatives when Logit-model has a linear form of utility function and has the same parameters of the utility function of AHP. Generally, this is linear mapping from the synthetic evaluation values of AHP to the utility values of Logit-model, and can be obtained by calculating the 
factor $k$ of the following equation, on the assumption that the choice probability of AHP and that of Logit-model become the same.

$$
\frac{\exp k W_{i}}{\sum_{j=1}^{m} \exp k W_{i}}-W_{i}=0 \quad(i=1,2, \cdots, m)
$$

As it is difficult to derive the factor $k$ directly, the logarithmic regression equation (where $m$ is the number of alternatives, $W_{\min }$ and $W_{\max }$ respectively are a maximum and a minimum value among the AHP values, and $\sigma$ is the standard deviation obtained from the analysis of the AHP values) is approximated in order to solve $k$. The obtained regression Eq. (2) is the coefficient of determination of 0.989630 and confirmed that there is no problem as the approximated equation.

$$
k=2.72536 m^{0.0500832} \sigma^{0.672027} W_{\max }^{-1.191172} W_{\min }^{0.004791}
$$

The discussion of the decision-making mechanism in AHP is not sufficient in comparison with one in Logit-model by this research. Therefore, both the research of the utility values about AHP and that of the decision-making mechanism seem to be necessary.

\subsection{Choice probability in AHP and utility function}

It is considered that the evaluation values (AHP values) of the weights in stratified AHP are the utility values. In other words, stratification structure is summarized in two levels about the alternative $x_{i}$. The evaluation value $W_{i}$ by AHP becomes the utility value when the weight of the criterion of the level 1 is equal to $w_{j}$ and the weight of the alternative of the level 2 is equal to $U_{j, i}$ as shown in Eq.(3).

$$
W_{i}=\sum w_{j} U_{j, i}
$$

AHP is one of the techniques for calculating the coefficient of the partial utility value when weight $w_{j}$ is to be determined, and AHP becomes one of the techniques for calculating utility value when $U_{j, i}$ is to be determined. When there are some alternatives, a decision-maker will choose the alternative most convenient to him/her. As the alternative of the large utility value is chosen in a choice opportunity as well, a proportional relation is thought to be established between the utility value and the choice probability. This choice probability is thought to show the rate at which an alternative is chosen, although it is sometimes different from the alternative of a maximum utility which is actually chosen even if the set of the same alternative is given. Therefore, the evaluation value of the alternatives by AHP is shown as a choice probability of the decision-maker to each alternative.

$$
P_{i}=\frac{W_{i}}{\sum_{j=1}^{m} W_{j}}
$$

The utility value $W_{i}$ can be expressed by Eq. (3) though this equation is the same type as Luce model ${ }^{(5)}$ whose utility value was not indicated clearly. The numerical value 1 through 9 is chosen in accordance with the prepared category (for example, in such cases as " same", " a little important", "very important") and the pairwise comparison is made with AHP. When an odd multiple is chosen based on a certain utility value, this operation approximates the ratio of the utility value of sincerity. Then, the relation between the utility value (evaluation value) $W_{i}$ obtained finally and the choice probability of alternatives are concluded to come to $P_{i}=k W_{i}$ in the interpretation with utility.

\subsection{Logit-model and utility function}

Logit-model, which McFadden has tied to the probabilistic utility theory, considers that the utility value of the alternatives is a random variable that changes irregularly, the change following the probability density function. Though the derivation of Logit-model with this probability density function is mathematical, it is not description of phenomenon in decision-making. So, a choice mechanism in decision-making is examined. Now suppose that a decision-maker selects one alternative from among the 
number of $m$ alternatives, who represents the group consisted of $N$ decision-makers. The following equation can be obtained from the sum of the choice probability becoming 1 about the choice probability $P_{i}$ which a certain alternatives $x_{i}$ is chosen.

$$
P_{1}+P_{2}+P_{3}+\cdots \cdots+P_{m}=1
$$

Before choosing either alternative, a decision-maker obtains some information from each alternative. The amount of information obtained from the alternative $x_{i}$ is described by using information entropy with $-\log P_{i}$, and the amount of information for decision-making can be shown as follows.

$$
H=-\sum_{i=1}^{m} P_{i} \log P_{i}
$$

Incidentally, if each alternative is uniform and there is no difference with the utility value, the amount of information with Eq. (6) becomes a maximum when the same choice probability is taken, and it is the same as when there is no information. However, there is a difference in the preference in the alternative, and the decision-maker is likely to give some meaning to the difference. Neumann has determined based on some axioms that the most rational principle for decision-making under uncertainty is the maximization of the expected value. This maximization of the expected value by Neumann is applied to the decision-making. Then let the utility value of the alternatives $x_{i} ; W_{i}$ and the choice probabilities make information entropy ${ }^{(6)}$ and expected value the maximum.

$$
L=-\sum_{i=1}^{m} P_{i} \log P_{i}+\alpha \sum_{i=1}^{m} W_{i} P_{i}+\beta\left(\sum_{i=1}^{m} P_{i}-1\right) \quad(\alpha, \beta \text { : undefined })
$$

The important and interesting equation can be led when the value obtained by differentiating the above equation in the choice probability $P_{i}$ is put with 0 .

$$
P_{i}=\exp (\beta-1) \cdot \exp \alpha W_{i}
$$

This equation defines the relations between the utility value $W_{i}$ and the choice probability $P_{i}$; choice probability is prescribed by utility value uniquely. It is shown by this equation that the probability chosen is large for an alternative with a large utility value. Logit-model (9) can be obtained when $\alpha W_{i}$ is put with $W_{i}$ and substituted for the Eq. (5).

$$
P_{i}=\frac{\exp W_{i}}{\sum_{j=1}^{m} \exp W_{j}}
$$

Therefore, it is clear that decision-making has the choice mechanism with maximum information entropy and maximum expected value. This derivation method proves the correctness of our intuitive and qualitative idea about decision-making.

\section{Discussion of AHP and Logit-model}

\subsection{Eigen value matrix in AHP}

The preceding section summarized stratification structure in two levels, and qualitatively described that the evaluation value $W_{i}$ of AHP becomes utility value and that utility value shows the choice probability. It has also been clarified that information entropy is a maximum in the choice probability of Logit-model as well as the expected value is a maximum. However, relations between AHP and the expected value and information entropy have not yet been explained. Evaluation with AHP is divided into the evaluation (the computation of the utility value) and the evaluation (the computation of the coefficient of the partial utility value) as shown in Eq. (3) when stratification structure is summarized in two levels. But, at any rate, AHP value is calculated by Eq. (10) defined by Saaty. 


$$
\left(\begin{array}{ccccc}
a_{1,1} & \cdots & a_{1, j} & \cdots & a_{1, n} \\
\vdots & \cdots & \vdots & \cdots & \vdots \\
a_{i, 1} & \cdots & a_{i, j} & \cdots & a_{i, n} \\
\vdots & \cdots & \vdots & \cdots & \vdots \\
a_{n, 1} & \cdots & a_{n, j} & \cdots & a_{n, n}
\end{array}\right)\left(\begin{array}{c}
w_{1} \\
\vdots \\
w_{j} \\
\vdots \\
w_{n}
\end{array}\right)=\left(\begin{array}{c}
w_{1} \\
\vdots \\
w_{j} \\
\vdots \\
w_{n}
\end{array}\right)
$$

Now, let's consider the case where the weight $w_{i}$ of the criteria is found when the evaluation value for a certain criterion is known. The eigen value matrix defined by Saaty assumes $a_{i, j}=1 / a_{j, i}$ and $a_{i, j}=w_{i} / w_{j}$, and does not consider that weight $w_{i}$ shows probability. Actually, each element of the matrix $\mathbf{A}$ is the relative comparison of the weight, and the action of choosing the numerical value of $1,3,5,7,9$ is not stochastic. In addition, an element $\left(a_{i, j}\right)$ can't be handled as a probability because the sum of each column or the sum of each line is not equal to1. It can be thought, however, that the first column of the criteria of matrix $\mathbf{A}$ is the first of $n$ criteria to evaluate in the beginning and that the second column of the criteria is the second to evaluate. If weight is utility value and a criterion is actually chosen at random at this time, the choice opportunity grows large as much as the criterion whose utility value is large, and the degree of the utility value shows the size of the choice probability. So, the probability of choosing the column $i$ in the criteria of the matrix $\mathbf{A}$ is expressed by $p_{i}$, and the probability of choosing the column $j$ in the criteria is expressed by $p_{j, i}$. Then, the following relation can be obtained when sum of each column in the Saaty's matrix is made 1.

$$
a_{j, i}=w_{j} / w_{i}=p_{j, i} / p_{i} \quad\left(\sum_{j=1}^{n} p_{j, i}=1, \sum_{j=1}^{n} p_{j}=1\right)
$$

At this time, the matrix $\mathbf{A}$ becomes the matrix $\mathbf{P}$ which has the elements with the conditional probability, and Eq. (10) is rewritten in the following equation.

$$
\left(\begin{array}{ccccc}
p_{1,1} & \cdots & p_{1, j} & \cdots & p_{1, n} \\
\vdots & \cdots & \vdots & \cdots & \vdots \\
p_{i, 1} & \cdots & p_{i, j} & \cdots & p_{i, n} \\
\vdots & \cdots & \vdots & \cdots & \vdots \\
p_{n, 1} & \cdots & p_{n, j} & \cdots & p_{n, n}
\end{array}\right)\left(\begin{array}{c}
p_{1} \\
\vdots \\
p_{j} \\
\vdots \\
p_{n}
\end{array}\right)=\left(\begin{array}{c}
p_{1} \\
\vdots \\
p_{j} \\
\vdots \\
p_{n}
\end{array}\right)
$$

Then, $a_{i, j} / \sum_{i=1}^{n} a_{i, j}=p_{i, j}$. If $p_{i, j}=p_{i}$, a column beside the matrix $\mathbf{P}$ is equivalent to the element of the eigen vector. In other words, it is shown that the eigen vector in the Saaty's matrix does not change even if the matrix $\mathbf{A}$ is changed into the matrix $\mathbf{P}$.

\subsection{Corelation between AHP and the Markov chain}

Though Eq. (10) and Eq. (12) show relations under the ideal conditions for decision-making, actual decision-making has uncertainty regarding alternatives and criteria, and may be done through various choice processes. Now, let the conditional stochastic matrix $\mathbf{P}$ describe $\mathbf{P}^{r}=\left(p_{i, j}{ }^{(r)}\right)$ when it goes through the choice process of the $r$ time, and the choice probability vector describe $\mathbf{p}^{r}$, Eq. (12) is shown as follows.

$$
\mathbf{p}^{r}=\mathbf{P p}^{r-1}
$$

$\mathbf{p}^{r}=\mathbf{P}^{r} \mathbf{p}^{0}$ can be taken if the stochastic matrix $P$ doesn't change. However, it has uncertainty along with the choice, and this uncertainty is indicated with information entropy. Now, if $\mathbf{p}^{0}$ is given and information entropy of $\mathbf{p}^{r}$ is made $H^{(r)}$, relations about the amount of information to make a decision are shown as follows.

$$
\begin{gathered}
0 \leq H^{(r)}-H^{(r-1)} \leq H^{(r-1)}-H^{(r-2)} \leq \cdots \cdots \leq H^{(1)}-H^{(0)} \\
H^{(r)}=H\left(\mathbf{p}^{r} \mid \mathbf{p}^{0}\right)
\end{gathered}
$$


In other words, the sequence $\left\{H^{(r)}\right\}$ of Eq. (14) has a convex by monotone increase, and the speed of the increase in entropy decreases gradually as choice processes increase. Uncertainty becomes the maximum at $H^{(r)}$, and little information is required to reach $H^{(r)}$ from $H^{(r-1)}$. When $r \rightarrow \infty, H^{(r)}$ becomes maximum, $\mathbf{p}^{r-1}, \mathbf{p}^{r} \rightarrow \mathbf{p}$ result, and Eq. (13) corresponds to Eq. (12). That is, making weight by AHP of the criteria can be handled as a choice probability by normalizing the sum of the column of the Saaty's matrix $\mathbf{A}$ in 1 where weight is presumed utility value. Then, it was showed that eigen vector in the eigen value matrix is the same as the vector where information entropy becomes maximum. It is said that information entropy is the maximum to make a decision, and showed that information entropy in AHP is the maximum.

\subsection{Maximization of the expected value}

It can be thought as well as 3.2 when evaluation $U_{j, i}$ is calculated by using AHP for a certain criterion, and the evaluation value $U_{j, i}$ is defined as the eigen vector to be maximum information entropy. If making weight by AHP is thought to be choice probability about the weight $w_{i}$, the right side of Eq.

(3) of the linear utility function shows expected value about the alternatives $x_{i}$. Generally, the utility function of $U_{j, i}$ is a convex form, and the theorem of Geoffrion; "At least one positive weight vector $w_{j}$ exists if utility becomes the maximum and utility is conversely the maximum if one positive weight vector $w_{j}$ exists", is applicable to the utility function as well. Because a choice probability vector $w_{j}$ about the criterion exists, utility value $W_{i}$ is composed of the expected value with AHP as indicated by Eq. (3), and so the utility is the maximum one. When the expected value was expressed as shown by Eq. (2), the following relation is derived with AHP.

$$
\sum_{i=1}^{m} P_{i} W_{i}=\sum_{i=1}^{m} W_{i}^{2}
$$

Therefore, it is shown that AHP whose expected value is maximized becomes the technique as well as Logit-model. And, when the synthetic evaluation value $W_{i}$ of AHP is taken, Eq. (15) proves that it becomes $k W_{i}$ the utility function of Logit-model that generally has a linear utility function form.

\section{Application to the measurement of the effect of information technology investment}

\subsection{An idea of the measurement}

Today, information technology investment in information technology, such as computer hardware and software, is large, and the organization behavior and business activities can't be conducted without information systems. And the quantitative evaluation of that effect has been very difficult. Parker et al ${ }^{(1)}$ showed a methodology to measure the effect in information technology investment for the first time, although their attempts are confined for the effect measurement from the subjective point of view. Although many researchers such as Paul Strassmann ${ }^{(3)}$ pointed out that the cost-benefit analysis should be applied to this kind of subjective evaluation method, no practical methodology has been presented.

On the basis of the systems of about fifty companies, we examined a framework about the effect measurement, and have determined that measurement of the effect of an information system is equal to measurement of service the system provide to users. Logit-model presented in the previous chapter is capable of handling the measurement of the service. However, Logit-model is influenced by a result of convergent operation of the logarithm likelihood function, and has a drawback that it cannot use many variables. Since AHP minimizes these problems with numerical operation, a technique for measuring the effect of the information system has been developed by using the agreement between AHP and Logit-model clarified in a preceding section. 


\section{2 Effect measuring model}

It is necessary to give a value directly with the value to the form-less criteria by using the utility value for the effect of an information system to be treated as the measurement of service to the user. So, let the utility value $W_{i}$ of the information system $i$ make the linear utility function which is the most popular form. Let the utility value for the criterion make $U_{j, i}$ and the weight make $w_{j}$, the effect value $W_{i}$ of the information system can be given with Eq. (3). Then, when the amount of information technology investment is made $B_{i}$, Eq. (3) is rewritten as follows.

$$
W_{i}=\sum_{j=1}^{n-1} w_{j} U_{j, i}-w_{n} B_{i}
$$

Both sides are divided with $w_{n}$ in this equation.

$$
W_{m i}=\sum_{j=1}^{n-1} w_{m j} U_{j, i}-B_{i}
$$

Then, the effect value $W_{m i}$ of Eq. (17) can be indicated by the amount of money, and $W_{m i}$ is caught with the amount of service from the information system $i$.In Eq. (17), the amount of money is ordinal utility value $U_{j, i}$, and the evaluation point is also ordinal utility value. Coefficient $w_{m j}$ shows the amount of partial ordinal utility value of one increment. Therefore, any kind of ordinal utility value scale can be made if it follows the rule that how to give the evaluation value of $w_{j}$ is fixed. It is possible to give a value directly with the value to the form-less criteria by using AHP. The size of the actual amount of service is found from the choice probability of the receptive person about the information systems of the $m$ individuals. In other words, the choice probability of the left side in Eq. (9) should be given, and $W_{i}$ is calculated by the degree of logarithm likelihood, and $W_{m i}$ is looked for from Eq. (17). But, all coefficients can't be always decided though a coefficient is looked for by using the logarithm likelihood function and by the official approval when the coefficient $w_{j}(j=1,2 \cdots, n-1)$ of Eq. (16) is looked for with Logit-model. At this time, AHP can be used for the evaluation of the criterion $w_{j}(j=1,2 \cdots, n-1)$ from the agreement of AHP and Logit-model.

\subsection{Determination of the coefficient of the linear utility function}

(1)Collection of evaluation data

Three information systems were selected from the information systems used by a certain traffic organization, and the information systems were prioritized in the order of effect determined by the questionnaire. The effect of an information system must be evaluated in a degree of achievement toward the goal value in a narrow sense; efficiency of the work, shortening the waiting time and rapid making of the project. But, grasping of the effect is difficult because desired value can't be established and because the influence of the effect is many-sided. So, for each information system, each of the following effect was evaluated and ranked from 1 to 9 .

Effect for human resource

Effect for information sharing

Effect for service

Effect for management

Effect for outside of an organization

(2)Evaluation of the attribute of the system

The criterion of each system was evaluated from the viewpoint of decision-maker, i.e., the user. An exponential ranking was used, since the evaluation by human is exponential as Lootsma ${ }^{(7)}$ pointed out(Table1).And, numerical values such as usual 1,3,5,7,9 were used for the comparison. The computation of the coefficient was executed by using the method of Lootsma (Table 1) and by using preference order of the information systems. As the information technology investment of around one person grows larger in the weight by usual 1,3,5,7,9, grows larger, or effect for human resource becomes a minus conversely. Evaluation value by the method of Lootsma can satisfy constraints about the sign. Incidentally, t-value of the effect for information sharing -the effect for outside of an organization can't 
be calculated, and Table 4-2 calculates only value.

Table 1. Evaluation of system attributes (Lootsma)

\begin{tabular}{l|c|c|c}
\hline Attributes & System A & System B & System C \\
\hline Total investment per person & $3,500,000$ yen & $1,500,000$ yen & $2,000,000$ yen \\
Effect for human resource & 8 & 16 & 11.3 \\
Effect for information sharing & 2.83 & 8 & 2 \\
Effect for service & 4 & 2 & 4 \\
Effect for management & 4 & 4 & 2 \\
Effect for outside & 2 & 1.41 & 4 \\
\hline
\end{tabular}

When the type of utility function is linear in AHP method, the authors have indicated the linear relationship between Logit-model and utility value with total utility value W. Then, the coefficient of all criteria can be calculated by AHP if either "the amount of investment around one person" or other qualitative criteria can be looked for. These coefficients are very small, and it seems to suggest that some significant conclusions couldn't obtain by the questionnaire. Table 4-3 shows the estimation results to each criterion by AHP based on the evaluation values by Lootsuma's method. Each coefficient is shown as follows.

Table 4-2. Evaluation of System Attributes (Logit-model)

\begin{tabular}{l|c|c|c}
\hline Attributes & $\begin{array}{c}\text { Parameter } \\
\text { Estimate }\end{array}$ & t-static & P-value \\
\hline Total investment per person & $-0.502858 \mathrm{E}-02$ & $0.994843 \mathrm{E}-02$ & -0.505465 \\
Effect for human resource & 0.537250 & 0.226805 & 2.36878 \\
Effect for information sharing & 0.016433 & 0. & 0. \\
Effect for service & 2.24813 & 0. & 0. \\
Effect for management & 0.560814 & 0. & 0. \\
Effect for outside & -0.349396 & 0. & 0. \\
\hline
\end{tabular}

Table 4-3. Evaluation by AHP

\begin{tabular}{l|c|c|c|c|c||l}
\hline & Human & Co-owning & Service & Management & Outside & $\begin{array}{l}\text { Eigen } \\
\text { Vector }\end{array}$ \\
\hline Human & 1 & 4 & 8 & 4 & 16 & 0.535459 \\
Co-owning & $1 / 4$ & 1 & 8 & 4 & 8 & 0.274994 \\
Service & $1 / 8$ & $1 / 8$ & 1 & $1 / 2$ & 4 & 0.055892 \\
Management & $1 / 4$ & $1 / 4$ & 2 & 1 & 8 & 0.111783 \\
Outside & $1 / 16$ & $1 / 8$ & $1 / 4$ & $1 / 8$ & 1 & 0.021873 \\
\hline
\end{tabular}

\section{Conclusion}

In this paper, on the assumption that evaluation value in AHP is the effect value, relations between AHP and Logit-model were examined using the information entropy that describes uncertainty. The latter half of the paper described the effect measurement technique developed on the basis of the relations between AHP and Logit-model obtained in the first half.

The results of these studies have led us to conclude as follows:

(1) From the utility value theory, it is reasonable to determine that the choice probability of an alternative in AHP is proportional to the ultimate utility value (evaluation value).

(2) The eigen vector in the Saaty's matrix does not change even when the sum of each column of the matrix is normalized to 1 .

(3) When a choice process is described by a model of Markov chain by normalizing the sum of each 
column of the Saaty's matrix, it is shown that information entropy of AHP is also maximized.

(4) Like a Logit-model, AHP is a decision-making tool that maximizes expected values, and the synthetic evaluation value is proportional to the linear form utility function of the Logit-model.

(5) There is a demand for measurement of the effect of the so-called information technology investment, that is, investment in computer hardware and software. A methodology for grasping this effect can be obtained from the relations between the Logit-model and AHP.

\section{Reference}

(1) Parker,M.M.\&Benson,Information Economics,Prentice Hall,Inc.,1988

(2) Synnnot,W.R.,The Information Weapon,John Wiley\&Sons,Inc.,1987

(3) Strassmann,P.A., The business Value of Computer,Information Economics Press, 1990

(4) Ozaki,T.,Kinoshita, E.\& Hara,L.,.Journal of Japan Society of Planning Civil Engineer, vol14,157-166,1997

(5) Luce,D.,Individual Choice Behavior, John Wiley\&Sons,Inc.,1959

(6) Shannon,C.E.,The Mathematical Theory Of Communication, The University of Illinois Press, 7-34,1959

(7) Lootsma,F.A., Scale Sensitivity and Rank Presentation in a Multiplicative Variant of the AHP, Delft University of Technology,1991 